experience is both well documented and scientifically pertinent.

Of course, scientists need to be rigorously objective too, which is why investigations are often reduced to a narrow focus. Although our English professor might accept the elimination of contextual variables, she would be most surprised by Frith's conviction that scientific enquiry can ultimately reveal "how the world truly is" through developing increasingly plausible models of it - the subject of a section in his final chapter.

Frith draws similarities between the brain's use of partial information to devise its world view and the practice of science itself. But if this is an interpretation based on illusion, does that not suggest that truth, too, is a fabrication, and is distorted by an anthropomorphic perspective? A glimpse at the history of science shows that models of how the physical world 'works' have often been debunked. Indeed, the discipline of neuroscience itself attracts a broad range of investigators - from researchers into artificial intelligence through to quantum theorists and panpsychists - whose suppositions about what 'works', and how, may be wildly different. One doesn't need to be a cultural relativist to desire a deeper level of discussion at this point.

Frith makes an innovative, if brief, specu- lation about the potential for a hermeneutics of neuroscience - that is, an investigation into the ways in which theories can be variously interpreted. His chapter is entitled 'How the Brain Creates Culture', but it is equally valid to ask to what extent culture creates the brain. Although some human perceptions and behaviours are undoubtedly hard-wired and autonomic, many others are acquired, not just in infancy when the brain is particularly plastic, but later in life too. The cultures we inhabit, besides the associations that we acquire as individuals, surely colour the ways in which we perceive the world - and how we form ideas about our mental processes.

Frith's approach confirms that our world picture is influenced by what Richard Gregory calls "the prevailing perceptual hypotheses", by different motivations, contexts and cognitive habits, and also, surely, by different cultural values and beliefs. This book proposes a subject that is ripe for investigation and it is one that might be productively shared by both sides, without drawing battle lines between those who are searching for one understanding of the mind and those who relish multiple explanations.

Siân Ede is arts director at the Gulbenkian Foundation, 98 Portland Place, London W1B 1ET. Her latest book is Art \& Science.

\title{
Two camps at cross-purposes
}

\section{Toxic Exposures: Contested IIInesses and the Environmental Health Movement by Phil Brown \\ Columbia University Press: 2007. 392 pp. $\$ 29.50$}

\section{Paul D. Blanc}

Reading Phil Brown's Toxic Exposures caused me to revisit C. P. Snow's seminal essay, The Two Cultures. Snow, writing almost 50 years ago, addressed what he perceived as a dangerous gulf between the world views of scientists and non-scientists. Snow's principal concern was how educational reform might bring the two camps closer. Although Brown is narrowly focused on selected human health effects (specifically breast cancer, childhood asthma and Gulf War illnesses) in relation to environmental exposure, his book implicitly concerns the ways in which scientists and non-scientists communicate and, more to the point, how they often speak at cross-purpose.

Brown comes to his subject matter from the social sciences, relying heavily on a qualitative approach informed by ethnography. $\mathrm{He}$ takes theoretical guidance from systematic critiques of the health-care system, such as Professional Dominance: The Social Structure of Medical Care (by Eliot Freidson) and The Social Transformation of American Medicine (by Paul Starr). Central to the thesis of Toxic
Exposures is a construct that Brown labels the 'dominant epidemiological paradigm'.

Brown intends this phrase to serve as useful shorthand for a conservative biomedical mindset that should be rejected, a mindset in which disease is narrowly seen as a matter of treatment and where causation is of interest (if it is taken into account at all) only insofar as

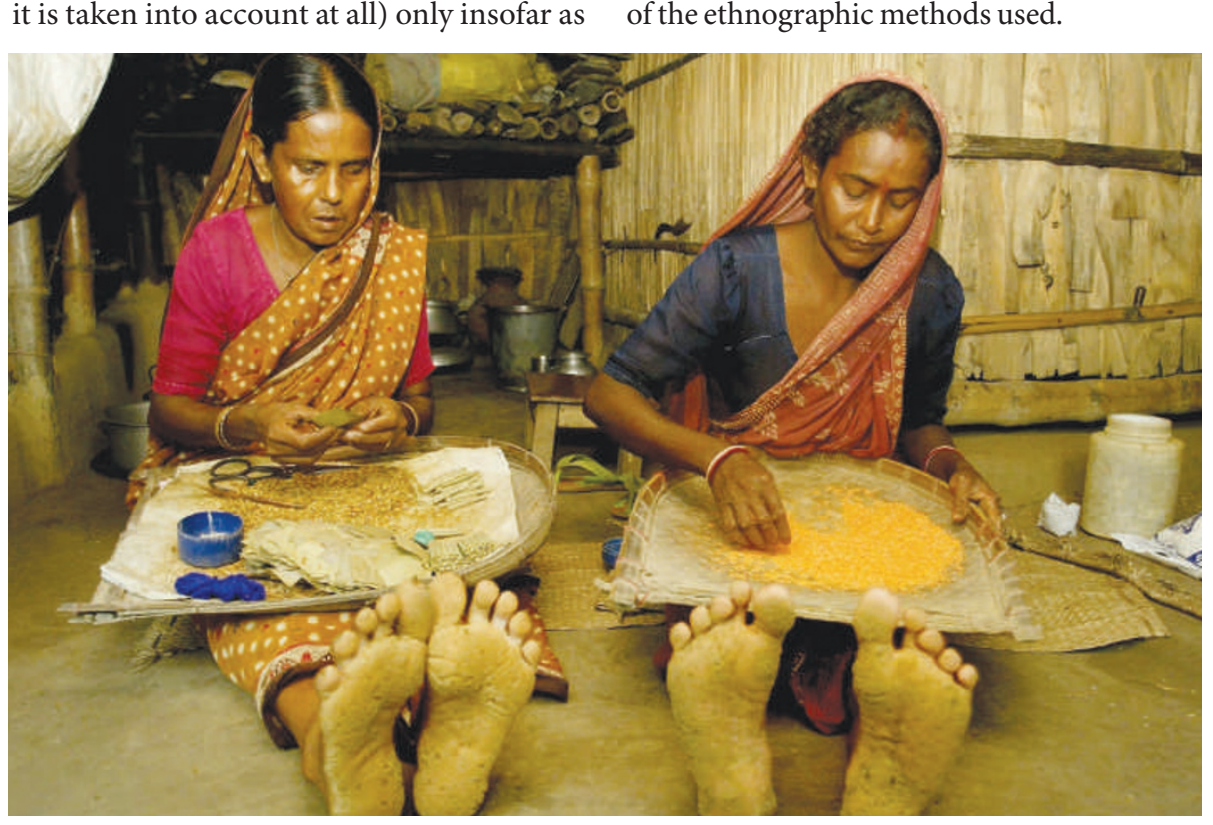

Arsenic-contaminated water affects hundreds of thousands of people in the Indian subcontinent. personal lifestyles are concerned. Here Brown may have put himself, and his readers, into a semantic double-bind. Epidemiology is inherently preoccupied with aetiology - the investigation of the causes and origin of a disease. The fairly recent emergence of 'clinical epidemiology' does indeed concern itself with treatments and outcomes, underscoring its differentiation from the parent discipline. Moreover, for most epidemiologists the supposition that personal lifestyle risk factors take exclusionary precedence over environmental factors remains to be established.

To support his argument, Brown takes a quite narrow view of what constitutes an environmental toxic exposure, basically limiting this to either an ambient air- or waterborne anthropogenic substance. Most epidemiologists are more catholic in their approach to defining the environment. Brown, for example, dismisses indoor air exposure as a valid environmental issue, without a convincing rationale for doing so. Indeed, he is explicitly critical of epidemiologists for being overly focused on indoor air quality. But this proscriptive approach does not even take into account second-hand cigarette smoke, one example of an indoor-air environmental exposure that is highly relevant both to asthma, and, as recent data suggest, breast cancer.

Toxic Exposures does shed light on the intersection of health research, advocacy and policy-making. Participatory research features prominently in this mix, although Brown does not purport to provide methodological guidance, and it would not be reasonable to expect this from his socio-ethnographic approach to the subject. (For a practical summary of core methods, see Social Epidemiology: Strategies for Public Health Activism by Julie Cwikel.) Nor should Brown be criticized for a geographical focus almost exclusive to the northeastern United States, given the logistical requirements of the ethnographic methods used. 
Fundamentally, however, I came away from Toxic Exposures confused as to its intended audience. The liberal use of didactic terms and constructs that presume fluency in the language and literature of medical sociology is likely to make the text too dense for most lay readers; there are also occasional passages that are overly doctrinaire and likely to put off even an otherwise receptive health scientist.
At one point in the text, Brown supports a seven-point manifesto of 'critical epidemiology', with the fifth point being: "Include in research reporting the explicit discussion of assumptions, values, and the social construction of scientific knowledge." This is certain to bewilder epidemiologists, most of whom see themselves as engaged scientists already bothered by a governmental policy-making apparatus that is overly politicized in the opposite direction. Fifty years on, it seems as though the two cultures are farther apart than ever. Paul D. Blanc is professor of occupational and environmental medicine at the University of California, San Francisco, California 94143-0924, USA and is the author of How Everyday Products Make People Sick: Toxins at Home and in the Workplace.

\section{Celebrating four centuries of astronomy}

\author{
The Telescope: Its History, Technology \\ and Future \\ by Geoff Andersen \\ Princeton University Press: 2007. 256 pp. \\ $\$ 29.95$
}

\section{René Racine}

The International Year of Astronomy in 2009 will mark 400 years of astronomical discovery, pioneered by Galileo Galilei in Padua, Italy. The anniversary will stimulate interest in the tools that made these discoveries possible, so a flush of new books on telescopes is likely to appear and sell briskly. Geoff Andersen's timely work also arrives at a point when astronomers and engineers are starting to construct a new generation of revolutionary instruments for ground-based and spacebased astronomy.

Andersen's book covers the historical and technological evolution of the telescope. It is written for a broad readership and "avoids, wherever possible, the gruesome details which keep so many of us employed". It will be appreciated by laypersons and experts for its clarity and, in places, the insight it provides into the optics, engineering and, indeed, scientific purposes and limitations of telescopes.

The book is enriched by the perspective it offers on remote sensing and surveillance applications, a field that the author has contributed to as a research physicist at the US Air Force Academy. This enables him to develop some interesting speculation on the capabilities of extant 'spy telescopes'.

Historical notes are exact, lively and generally up-to-date. Witness the recent discovery of the full name of Laurent Cassegrain - seventeenth-century inventor of the Cassegrain telescope - uncovered by André Baranne and Françoise Launay in the Journal of Optics in 1997, and not generally known even to experts. References to current projects, such as the forthcoming Hubble Space Telescope service mission, the Thirty Meter Telescope partnership and the 100-metre-aperture OWL (for 'overwhelmingly large') telescope, are inevitably dated, given the rapid evolution of the field.

Professional astronomers and experienced amateur telescope-makers might be tempted to skip the introductory chapters on optics,

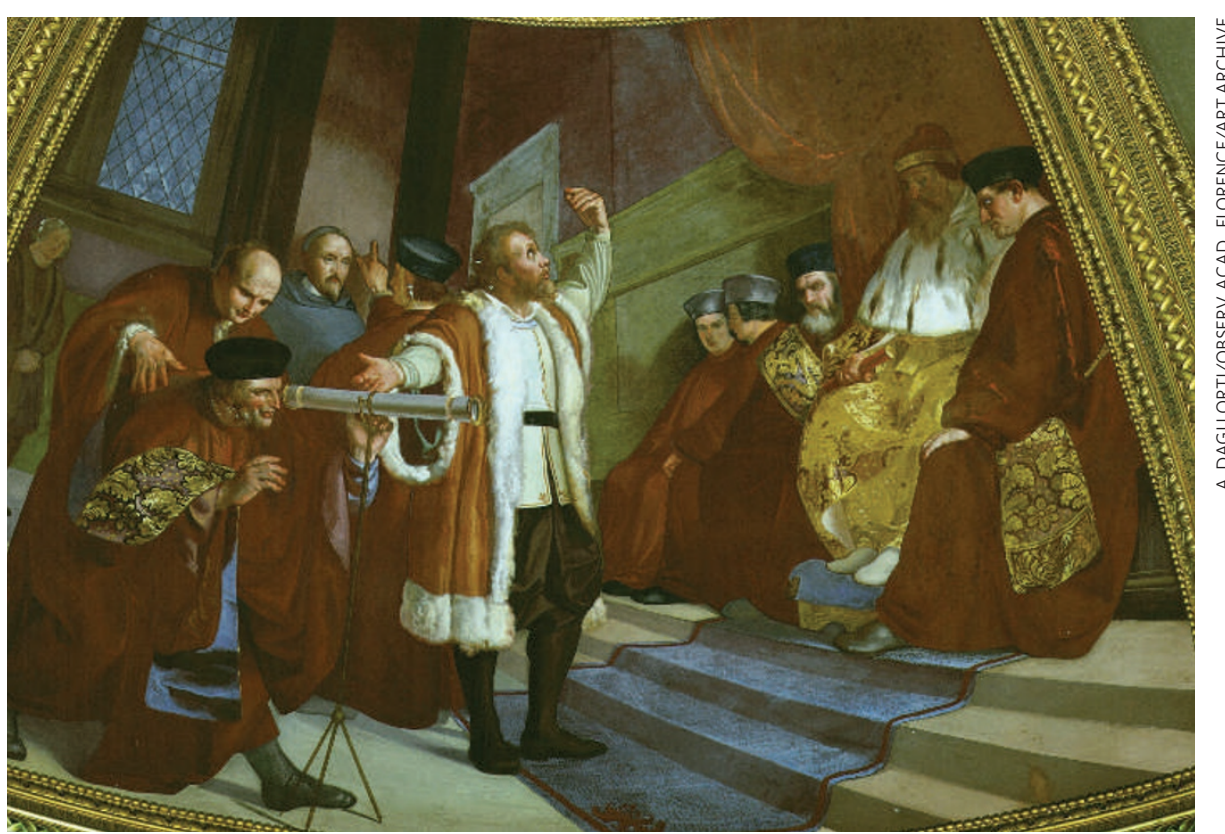

Galileo presents the most powerful telescope of its time to the Doge and Senate of Venice in 1609.

the basics of telescope design, light-analysis instruments and observing-site properties, but it would be at the cost of missing some pedagogical gems. These include the quasiintuitive explanation of Poisson's 'bright spot', which vindicated the theory of diffraction and the wave nature of light.

Informative chapters on interferometry and advanced telescope techniques - active and adaptive optics, laser guide stars, laser communications, and so on - are seldom found in such detail in similar works, adding to the value of the book. The analogies and differences in telescopes operating in various domains, from $\gamma$-rays to radio waves and even to gravitational waves, are nicely presented and help in understanding how some more exotic instruments operate.

An author's view of a field is necessarily influenced by background and experience. Hence, Andersen's assertion that "In 1991 the US Air Force decided to declassify the existence of adaptive optics. Overnight, the field of astronomy took a giant leap forward unlike any since the development of mirrored optics" might have been couched differently by civilian French and American astronomers and by technologists who independently developed highly productive astronomical adaptive systems in the 1980s. Likewise, some might question his estimate that "Military space technology tends to be 10-20 years ahead of that in the civilian world".

In a quite different vein, the author's opinion that "Antoine de Saint-Exupéry's accomplishments [as a surveillance pilot] are overshadowed by a children's book he wrote which is widely used to take the fun out of learning French" will sadden many who have been charmed by Le Petit Prince - and it is not relevant to the understanding of telescopes by laypersons.

Andersen's fine piece of work is enlightening and interesting. Experts can dispense with a few chapters that beginners will find useful, along with the appendices on mathematical notation, electromagnetic radiation and "Getting your own telescope". The text flows smoothly, enhanced by vivid discussion and pedagogical flair. And the author's take on remote sensing and surveillance confers a unique feature that will fascinate readers.

René Racine is professor emeritus in the Department of Physics at the Université de Montréal, PO Box 6128, Station Centre-Ville, Montréal, Québéc H3C 3J7, Canada. 